\title{
Chirped Gratings in Integrated Optics
}

\author{
A. KATZIR, A. C. LIVANOS, J. B. SHELLAN, AND A. YARIV
}

\begin{abstract}
Gratings with variable periods (chirped gratings) have been fabricated by recording the interference pattern of a collimated laser beam with a converging beam generated by a cylindrical lens. An analysis is presented for the behavior of the chirped gratings as a function of wavelength, the angle between the illuminating beams, the $F$ number of the lens, and its position. To calculate the power radiated into air, the coupled-mode equations are solved for the case of a waveguide with chirped surface corrugation. Experimentally, chirped gratings have been etched on the surface of an optical waveguide and used to couple light out of the waveguide. It was found that the light was focused outside the waveguide, and the fraction of the power radiated into air compared favorably with the theoretical calculation. The focal point outside the waveguide was found to move by about $1 \mathrm{~cm}$ when the wavelength was changed by $500 \AA$-in agreement with theoretical estimates.
\end{abstract}

\section{INTRODUCTION}

$\mathrm{P}$ ERIODIC STRUCTURES, and in particular corrugated structures, play a significant role in integrated optics [1]. Corrugated waveguides serve as narrow-band filters, which reflect wavelengths which satisfy Bragg's law [2]. Such reflectors may be incorporated in laser structures to form distributed feedback lasers [3] or distributed Bragg reflectors [4]. Periodic structures with longer periods have been used to couple between guided modes and air, such as in the cases of input or output couplers [5] .

In this paper, we consider the problem of gratings with large and monotonic variation in the period. We describe a method for fabricating such chirped gratings, present a theory for treating them, and present experimental results demonstrating some of their unique applications.

\section{Grating Fabrication Considerations}

The gratings are fabricated, as in the case of uniform gratings, by the interference of two laser beams. The period chirp is obtained by cylindrical focusing of one of the two beams, as shown in Fig. 1. The recording plate is located at the $x=0$ plane, the angle of incidence of the plane wave is $\theta / 2$, and the angle subtended by the collimated beam and the bisector of the converging beam angle is $\theta$. The interference pattern is recorded over a distance $L$ on the recording plate. The converging wave is generated by a cylindrical lens of focal length $f$ and width $d$, and the focus is located at point $P\left(x_{f}, z_{f}\right)$.

Simple geometrical calculations relate the focal line coordinates with $f, L, d$, and $\theta$, namely

Manuscript received November 16, 1976; revised December 16, 1976. This research was supported by AFOSR. The work of one of the authors ( J. B. S.) was supported in part by the Hertz Foundation.

The authors are with the California Institute of Technology, Pasadena, CA 91125 .

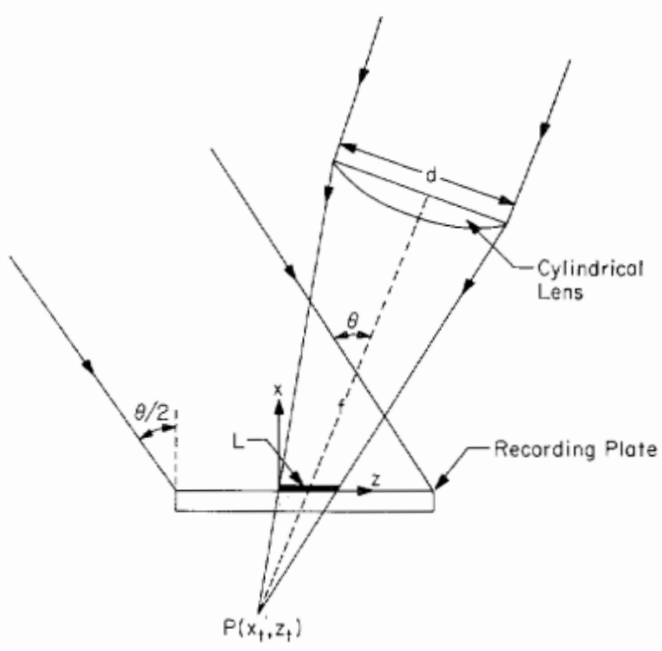

Fig. 1. Recording arrangement and geometry for the fabrication of chirped gratings.

$$
x_{f}=\frac{-L \cos \left(\phi+\frac{\theta}{2}\right)}{2 \sin 2 \phi} \cos \left(\phi-\frac{\theta}{2}\right)
$$

and

$$
z_{f}=\frac{L \cos \left(\phi+\frac{\theta}{2}\right)}{\sin 2 \phi} \sin \left(\phi-\frac{\theta}{2}\right)
$$

where

$$
\phi=\tan ^{-1}\left(\frac{d}{2 f}\right)
$$

is the convergence half-angle. We note that in (1) $x_{f}$ is always negative, while $z_{f}$ can take negative or positive values depending on the angles $\theta$ and $\phi$.

The electric field in the recording plane $(x=0)$ is given by the sum of the reference wave and converging one and is given by

$$
\begin{aligned}
E(x=0, z)= & A \exp -i k z \sin (\theta / 2) \\
& +\alpha \exp i k\left\{\left[\left(z-z_{f}\right)^{2}+x_{f}^{2}\right]^{1 / 2}\right\}
\end{aligned}
$$

where $k=2 \pi / \lambda$ is the wavenumber for the incident field, and $A$ and $\alpha$ are the amplitudes of the plane and converging wave, respectively. If we assume that the transmission function of the recording medium $t$ is proportional to $E E^{*}[6]$, and that $A=\alpha$, then

$$
t=\beta\left[1+\cos \left\{k z \sin (\theta / 2)+k \sqrt{\left(z-z_{f}\right)^{2}+x_{f}^{2}}\right\}\right]
$$

where $\beta$ is a proportionality constant. The period $\Lambda$ for this particular grating is given by 


$$
\Lambda(z)=\frac{\lambda}{\sin (\theta / 2)+\frac{z-z_{f}}{\sqrt{\left(z-z_{f}\right)^{2}+x_{f}^{2}}}} .
$$

In the paraxial approximation $\left(z-z_{f}\right)^{2}<<x_{f}^{2}$, (4) and (5) reduce to

$$
\begin{aligned}
t= & \beta\left[1+\cos \left\{\frac{k}{2 x_{f}} z^{2}+\left(k \sin (\theta / 2)-\frac{k z_{f}}{x_{f}}\right) z\right.\right. \\
& \left.\left.+k x_{f}+\frac{k z_{f}^{2}}{2 x_{f}}\right\}\right] .
\end{aligned}
$$

The corresponding expression for (5) is therefore

$$
\Lambda(z)=\frac{\lambda}{\sin (\theta / 2)+\left(z-z_{f}\right) / x_{f}} .
$$

It is seen from (1), (2), and (5) that the period variation $\Lambda(z)$ depends on the $F$ number of the lens $(F \equiv f / d)$, and that $\theta$ is the angle subtended by the collimated beam and the bisector of the converging beam angle, $\lambda$ is the wavelength of illumination, and $L$ is the length of the grating.

The dependence of the period variation on $F$ is illustrated by Figs. 2 and 3 . In Fig. 2, the angle $\theta$ is set at $60^{\circ}$ and the grating has a total length of $1 \mathrm{~cm}$. For various $F$ numbers, period variations from $0.8 \mu \mathrm{m}$ to $0.4 \mu \mathrm{m}$ are obtained. The lower the $F$ number the greater the period variation; higher $F$ numbers result in smaller and more linear period variations. In Fig. 3, the angle $\theta$ has the value of $90^{\circ}$. Here the maximum period variation is for an $F=1$ lens and it extends from 0.45 to $0.28 \mu \mathrm{m}$ over a distance of $1 \mathrm{~cm}$. It is noted that large values of $\theta$ produce smaller period variations.

This particular point is illustrated in Fig. 4, where an $F=$ 1.33 lens was chosen and $\theta$ was varied from $45^{\circ}$ to $120^{\circ}$. Again the grating extends over a distance of $1 \mathrm{~cm}$. It is seen that with $\theta=120^{\circ}$, the period varies only by $0.05 \mu \mathrm{m}$, while for $\theta=45^{\circ}$ the period variation is $0.5 \mu \mathrm{m}$.

The linearity of the period variation as a function of grating length $L$ is shown in Fig. 5. It should be noted that the beginning and end period is identical for all values of $L$. Again the $F$ number is 1.33 and the angle $\theta$ is $90^{\circ}$.

\section{WAVEguide Coupling}

Chirped grating etched onto a dielectric waveguide results in a simultaneous output coupling and focusing to a point $P\left(x_{\lambda}, z_{\lambda}\right)$, which will vary as a function of the modes supported by the waveguide and the wavelength of the guided modes.

Consider the geometry described by Fig. 6 . When the guided mode is propagating unpertrubed in the waveguide, its $z$ dependence is given by $e^{-i \beta z}$, where $\beta=k n_{1} \cos \theta_{1}$. When the wave reaches the perturbation, the radiated mode will have a $z$ dependence given by $e^{-i k_{z} z}$. At point $z=0, k_{z}$ is given by

$$
k_{z}(0)=\beta-\frac{2 \pi}{\Lambda(0)}
$$

and at $z=L$

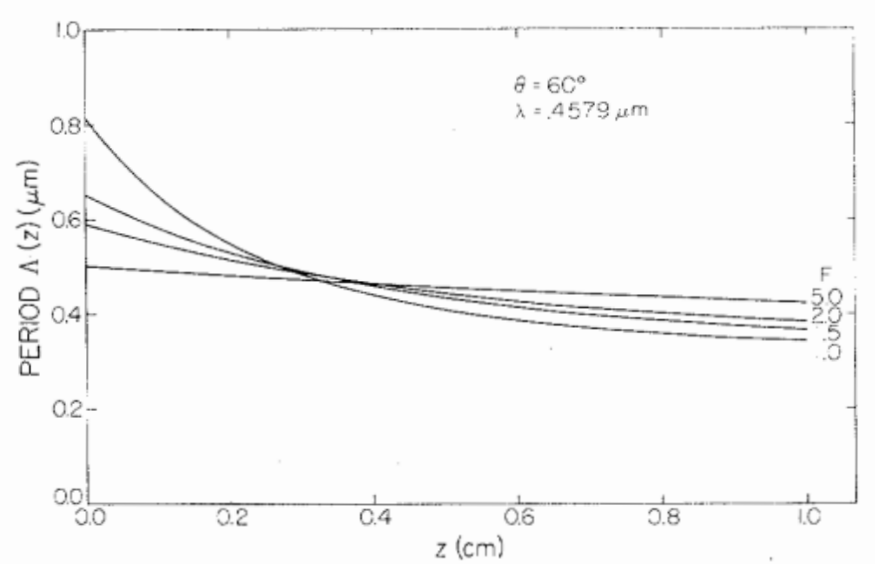

Fig. 2. Period variation as a function of the $F$ number of the converging lens $(F=f / d)$. The angle $\theta=60^{\circ}$ is suitable for variations of $0.8-0.4 \mu \mathrm{m}$ over a distance of $1 \mathrm{~cm}$.

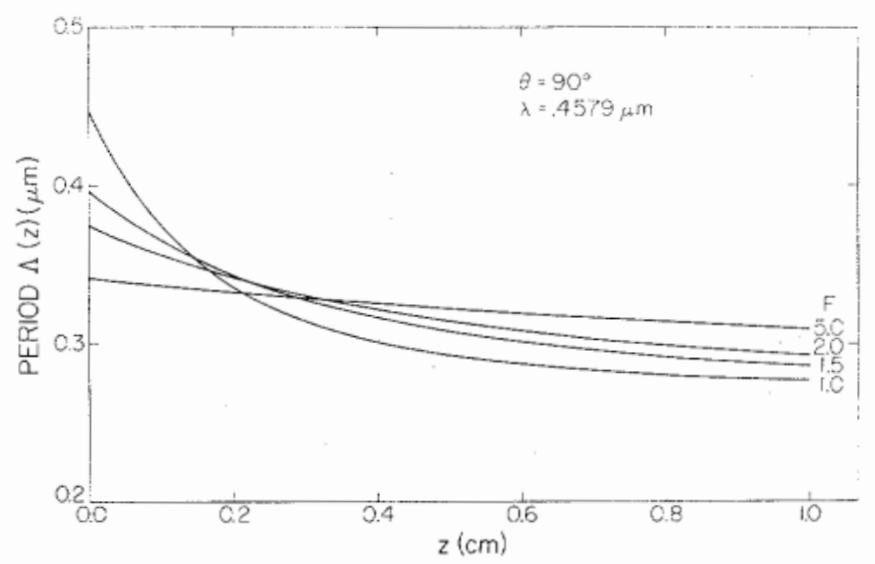

Fig. 3. Period variation as a function of the $F$ number. The angle $\theta$ has a value of $90^{\circ}$ and the range of period variation is from 0.45 to $0.28 \mu \mathrm{m}$, again over a distance of $1 \mathrm{~cm}$.

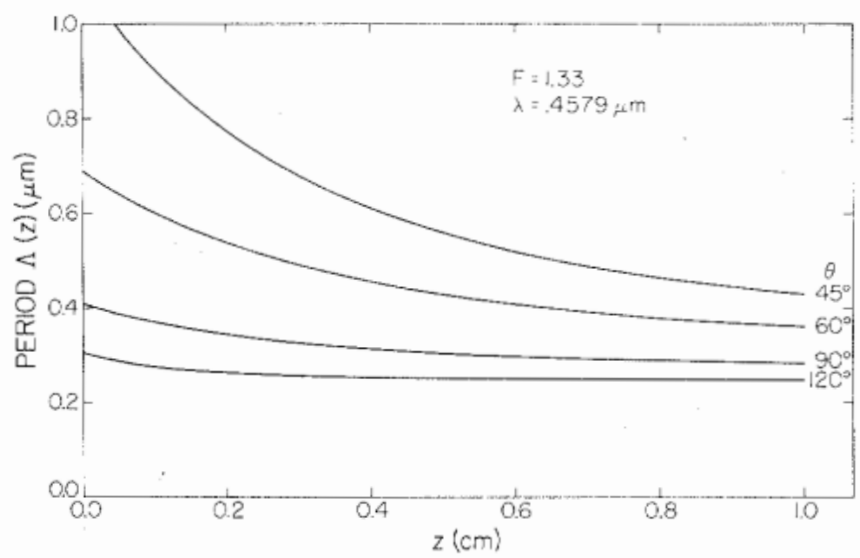

Fig. 4. Period variation as a function of $\theta$ (the angle between the plane wave and the bisector of the converging wave). The $F$ number of the lens is 1.33 and the illumination wavelength is $0.4579 \mu \mathrm{m}$. The recording distance is kept constant at $1 \mathrm{~cm}$.

$$
k_{z}(L)=\beta-\frac{2 \pi}{\Lambda(L)} .
$$

It can be shown that by a) matching the tangential component of the electric field inside and outside the waveguide, b) requiring that $k^{2}=k_{x}^{2}+k_{z}^{2}$ outside the waveguide, and c) 


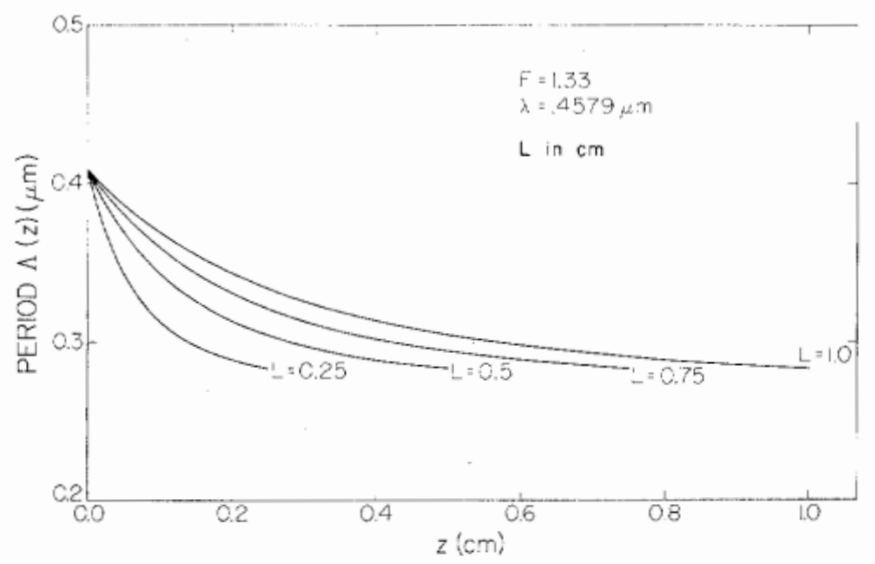

Fig. 5. Period variation as a function of recording distance $L$. The total amount of chirp is the same for all curves; the linearity of variation is seen to improve for large values of $L$. The angle $\theta$ is $90^{\circ}$, the $F$ number is 1.33 , and the wavelength is $0.4579 \mu \mathrm{m}$.

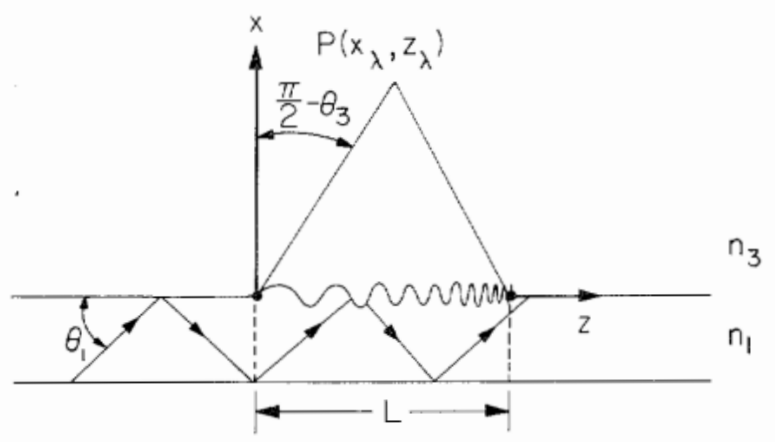

$n_{2}$

Fig. 6. Geometry for a chirped grating etched on the top surface of a waveguide of index $n_{1}$. The substrate has an index $n_{2}$, and $n_{3}$ in the index of refraction of air. A waveguide mode will focus at point $P\left(x_{\lambda}, z_{\lambda}\right)$, depending on the chirp of the grating and the wavelength.

assuming that the transmission function for the grating is given by (4), the light will focus outside at a point $P\left(x_{\lambda}, z_{\lambda}\right)$ given by

$$
z_{\lambda}=\frac{k_{z}(0) L \sqrt{k^{2}-k_{z}^{2}(L)}}{k_{z}(0) \sqrt{k^{2}-k_{z}^{2}(L)}-k_{z}(L) \sqrt{k^{2}-k_{z}^{2}(0)}}
$$

and

$$
x_{\lambda}=\frac{\sqrt{k^{2}-k_{z}^{2}(0)}}{k_{z}(0)} z_{\lambda} .
$$

The focusing effect and especially the variation of the focus as function of wavelength and period variation is illustrated by Fig. 7. Taking $n_{1}=1.565, n_{2}=1.51, n_{3}=1.0$, and a waveguide thickness of $d=1.35 \mu \mathrm{m}$, the eigenvalue equation for $\beta$ was solved for wavelengths ranging from 4500 to $6500 \AA$. Having thus determined $\beta$ for the unperturbed waveguide, we calculate $k_{z}(0), k_{z}(L)$ for various ranges of period variation. It can be seen from this figure that a) the larger the period variation the closer to the waveguide the locus of the focal points will be, b) the smaller the period variation the larger

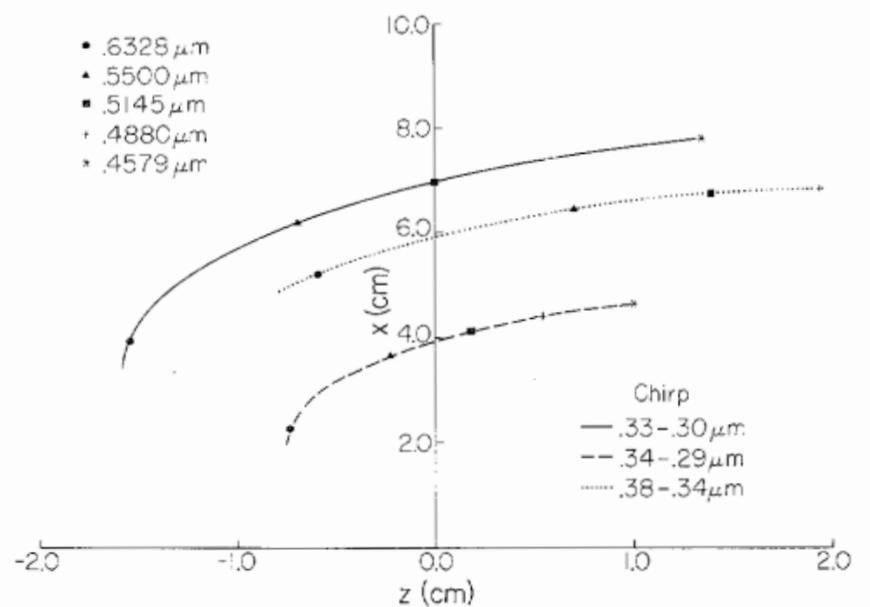

Fig. 7. Locus of the foci of various wavelenghts for different chirps. The grating is located between $z=0$ and $z=1.0 \mathrm{~cm}$ at $x=0 . \Lambda(0)$ is the longest period and $\Lambda(1 \mathrm{~cm})$ is the shortest. The waveguide mode is traveling in the positive $z$ direction.

the separation between the different wavelengths and the larger the distance of the locus of the focal points from the waveguide, and c) if the average period of the chirped grating is increased the focus will shift towards greater values of $z$.

\section{Calculation of Power Output Distribution For Chirped Gratings}

In the previous section we discussed the characteristics of the chirped gratings and some of their properties. To complete our theoretical discussion we present a calculation of the actual power radiated into air by a chirped grating.

To analyze this problem we expand the electric field of the perturbed waveguide in terms of the guided modes, the substrate modes, and the air modes. This work is essentially an extension of Marcuse's work [7], in so much that in our case the waveguide is no longer symmetric (we include the substrate). Our notation and method are similar to his.

We present a closed-form solution for the power radiated into air by a chirped grating, and illustrate the solution with examples of gratings where we vary the amount of chirp and the wavelength of the guided radiation.

Consider the geometry and notation as presented in Fig. 8(a). Using the results obtained by Marcuse [8], we have for the TE guided modes

$$
\begin{aligned}
\mathscr{E}_{y} & =A e^{-\delta x}, & & \text { for } x \geqslant 0 \\
& =A\left[\cos \kappa x-\frac{\delta}{\kappa} \sin \kappa x\right], & & \text { for } 0 \geqslant x \geqslant-d \\
& =A\left[\cos \kappa d+\frac{\delta}{\kappa} \sin \kappa d\right] e^{\gamma(x+d)}, & & \text { for } x \leqslant-d
\end{aligned}
$$

where

$$
\begin{aligned}
& \kappa \equiv\left(n_{1}^{2} k^{2}-\beta^{2}\right)^{1 / 2} \\
& \gamma \equiv\left(\beta^{2}-n_{2}^{2} k^{2}\right)^{1 / 2} \\
& \delta \equiv\left(\beta^{2}-n_{3}^{2} k^{2}\right)^{1 / 2}
\end{aligned}
$$




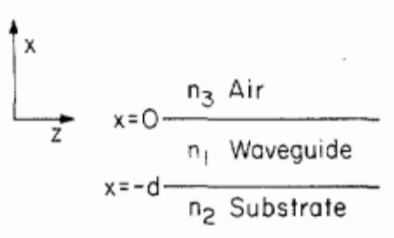

(a)

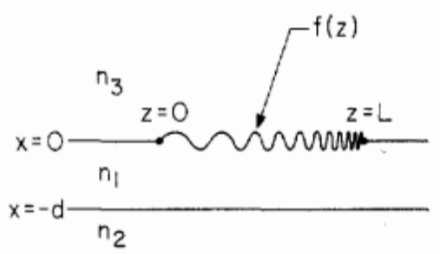

(b)
Fig. 8. (a) Geometry for a dielectric waveguide. (b) Dielectric waveguide with a chirped grating etched on the top surface.

where $n_{1}, n_{2}, n_{3}$ are the indices of refraction of the waveguide, substrate, and air, respectively, $k$ is the wavenumber in air, and $\beta$ describes the $z$ dependence of the electric field. It should be noted that the factor $e^{i \omega t} e^{-i \beta z}$ has been suppressed in (12)-(14). Furthermore, the constants $\kappa, \gamma$, and $\delta$ can be determined by the eigenvalue equation

$$
\tan \kappa d=\frac{\kappa(\gamma+d)}{\kappa^{2}-\gamma \delta} .
$$

The amplitude of the electric field $A$ is related to the power carried by the mode, namely

$$
\begin{aligned}
A^{2} & =\frac{4 \kappa^{2} \omega \mu_{0} P}{|\beta|[d+1 / \gamma+1 / \delta]\left(\kappa^{2}+\delta^{2}\right)} \\
P & =\frac{\beta}{2 \omega \mu_{0}} \int_{-\infty}^{\infty}\left|\varepsilon_{y}\right|^{2} d x
\end{aligned}
$$

where $P$ is the power carried by the mode, $d$ is the thickness of the guide, $\omega$ is the radian frequency, and $\mu_{0}$ is the magnetic permeability of vacuum.

These guided modes occur for $k n_{2} \leqslant|\beta| \leqslant k n_{1}$. For the region $k n_{3} \leqslant|\beta| \leqslant k n_{2}$ the substrate modes exist, and, finally, in the region $0<|\beta|<k n_{3}$ the TE air modes of the continuum occur. For the purposes of this discussion we consider the air mode since we want to calculate the power radiated by the waveguide into the air. Reference [8] gives the electric field as

$$
\begin{aligned}
\stackrel{8}{a}_{y}^{a}= & C_{r}\left[\cos \Delta x+(\sigma / \Delta) F_{i} \sin \Delta x\right] \\
= & C_{r}\left(\cos \sigma x+F_{i} \sin \sigma x\right) \\
& \text { for } x \geqslant 0 \\
= & C_{r}\left[\left(\cos \sigma d-F_{i} \sin \sigma d\right) \cos \rho(x+d)\right. \\
& \left.+\frac{\sigma}{\rho}\left(\sin \sigma d+F_{i} \cos \sigma d\right) \sin \rho(x+d)\right], \\
& \quad \text { for } x \leqslant-d \\
\Delta= & \left(n_{3}^{2} k^{2}-\beta^{2}\right)^{1 / 2} \\
\sigma= & \left(n_{1}^{2} k^{2}-\beta^{2}\right)^{1 / 2} \\
\rho= & \left(n_{2}^{2} k^{2}-\beta^{2}\right)^{1 / 2}
\end{aligned}
$$

where $C_{r}$ is again related to the power carried by the mode

$$
C_{r}^{2}=\frac{4 \omega \mu_{0} P}{\pi|\beta|}\left[\left(\cos \sigma d-F_{i} \sin \sigma d\right)^{2}\right.
$$

$$
\begin{aligned}
& +\frac{\sigma^{2}}{\rho^{2}}\left(\sin \sigma d+F_{i} \cos \sigma d\right)^{2} \\
& \left.+\left(1+\frac{\sigma^{2}}{\Delta^{2}} F_{i}^{2}\right) \frac{\Delta}{\rho}\right]^{-1}
\end{aligned}
$$

and $F_{i}$ can be chosen arbitrarily. Following the conventional procedure, $F_{1}$ and $F_{2}$ are chosen so that the two radiation modes are orthogonal to one another.

$$
\begin{aligned}
F_{1,2}= & {\left[\left(\sigma^{2}-\rho^{2}\right) \sin 2 \sigma d\right]^{-1}\left\{\left(\sigma^{2}-\rho^{2}\right) \cos 2 \sigma d\right.} \\
& +\left(\frac{\rho}{\Delta}\right)\left(\sigma^{2}-\Delta^{2}\right) \pm\left[\left(\sigma^{2}-\rho^{2}\right)^{2}\right. \\
& +2(\rho / \Delta)\left(\sigma^{2}-\rho^{2}\right)\left(\sigma^{2}-\Delta^{2}\right) \cdot \cos 2 \sigma d \\
& \left.\left.+\left(\rho^{2} / \Delta^{2}\right)\left(\sigma^{2}-\Delta^{2}\right)^{2}\right]^{1 / 2}\right\}
\end{aligned}
$$

where

$$
P \delta\left(\rho-\rho^{\prime}\right)=\frac{\beta^{*}}{2 \omega \mu_{0}} \int_{-\infty}^{\infty} \hat{\mathscr{E}}_{y}^{a}(\rho) \hat{\mathscr{E}}_{y}^{a *}\left(\rho^{\prime}\right) d x .
$$

Again the factor $e^{i \omega t} e^{-i \beta z}$ has been suppressed in (20)(22). In this work $\beta$ is an inherently positive quantity.

Next we expand an arbitrary TE electric field for the perturbed waveguide in terms of the discrete guided modes and the continuum of both substrate and air modes

$$
\begin{aligned}
E_{y}= & \sum_{\text {discrete }} C_{n}(z) \varepsilon_{n}+\int_{0}^{k\left(n_{2}^{2}-n_{3}^{2}\right)^{1 / 2}} g(\rho, z) \&^{s}(\rho) d \rho \\
& +\sum_{\substack{\text { even } \\
\text { odd }}} \int_{k\left(n_{2}^{2}-n_{3}^{2}\right)}^{k n_{3}} h(\rho, z) \&^{a}(\rho) d \rho
\end{aligned}
$$

where $\mathscr{E}_{n}$ are the discrete guided modes given by (12)-(14) for the $n$ values of $\beta$ determined from the eigenvalues of (18). Similarly, $\&^{a}$ are the air modes given again by (20)-(22), where even and odd refer to the choice of $F_{2}$ and $F_{1}$ (27). $\varepsilon^{s}$ are the substrate modes which have not been presented explicitly since they do not affect this calculation. It is to be noted that the previous expansion for the total electric field $E_{y}$ is possible since the set of eigenfunctions is complete. The calculation is simplified due to the orthogonality of the modes as a result of the choice of $F_{i}$. Furthermore, this expansion is similar to the one present in [7], as well as the notation and the method used to solve this problem.

To determine the value of $h(\rho, z)$, we substitute (28) into the Helmholtz wave equation, multiply by $\varepsilon^{* a}$, integrate over $x$, and, using the orthogonality relations, get a differential equation for $h(\rho, z)$. This differential equation is then converted into an integral equation following the procedures of [7], namely

$$
\begin{aligned}
h(\rho, z)= & Q(\rho)+R(\rho) \exp 2 i \beta z+\frac{1}{2 i \beta} \\
& \cdot \int_{0}^{z}[\exp 2 i \beta(z-\zeta)-1] H(\rho, \zeta) d \zeta
\end{aligned}
$$

where 


$$
\begin{aligned}
H(\rho, z)= & \frac{-\beta k^{2}}{2 \omega \mu P}\left[\sum_{n} C_{n}(z) \int_{-\infty}^{\infty} \varepsilon^{* a}(\rho) \Delta n^{2} \varepsilon_{n} d x\right. \\
& +\int_{k\left(n_{2}^{2}-n_{3}^{2}\right)^{1 / 2}}^{k n_{2}} d \rho^{\prime} g\left(\rho^{\prime}, z\right) \int_{-\infty}^{\infty} \varepsilon^{* a}(\rho) \\
& \cdot \Delta n^{2} \&^{s}\left(\rho^{\prime}\right) d x+\int_{k\left(n_{2}^{2}-n_{3}^{2}\right)^{1 / 2}}^{k n_{2}} d \rho^{\prime} h\left(\rho^{\prime}, z\right) \\
& \cdot \int_{-\infty}^{\infty} \varepsilon^{* a}(\rho) \Delta n^{2} \varepsilon^{a}\left(\rho^{\prime}\right) d x
\end{aligned}
$$

where $\Delta n^{2}$ describes the deviation of the corrugated-guide dielectric constant from that of a uniform waveguide.

To solve the previous integral equation, we use the Born approximation. In other words, we use $C_{n}(0)=\delta_{0 n}$ instead of $C_{n}(z)$ and set $g(\rho, z)=h(\rho, z)=0$, in (30), resulting in

$$
H(\rho, z) \simeq \frac{-\beta k^{2}}{2 \omega \mu P} \int_{-\infty}^{\infty} \varepsilon^{* a}(\rho) \Delta n^{2} \S_{0} d x .
$$

In the next step, we assume that the perturbation of the guide from its ideal shape is on the top surface of the guide, as shown in Fig. 8(b). By taking a shallow grating and setting $x=0$ in the previous equation, we get

$$
H(\rho, z)=\frac{-\beta k^{2}}{2 \omega \mu P}\left(n_{1}^{2}-n_{3}^{2}\right) f(z) \varepsilon^{* a}(0, \rho, z) \varepsilon_{0}(0, z) .
$$

Equation (29) can then be divided into parts as follows:

$$
\begin{aligned}
& h^{+}=Q-\frac{1}{2 i \beta} \int_{0}^{z} H(\rho, \zeta) d \zeta \\
& h^{-}=\left[R+\frac{1}{2 i \beta} \int_{0}^{z} \exp -2 i \beta \zeta H(\rho, \zeta) d \zeta\right] \exp 2 i \beta z
\end{aligned}
$$

such that

$$
h=h^{+}+h^{-} .
$$

Recalling (28), we note that the contribution to the total electric field arises from the product of $h(\rho, z) \cdot \mathscr{E}^{a}(\rho, z)$. The $z$ dependence of $\varepsilon^{a}(\rho, z)$ is $e^{-i \beta z}$. If we consider the $z$ dependence of the product, then

$$
\begin{aligned}
\underset{(z \text { dependence })}{h(\rho, z) \mathbb{E}^{a}(\rho, z)=} & h^{+} \exp -i \beta z+\left[R+\frac{1}{2 i \beta}\right. \\
& \left.\cdot \int_{0}^{z} \exp -2 i \beta \zeta H(\rho, \zeta) d \zeta\right] \exp i \beta z .
\end{aligned}
$$

Then we can associate the $h^{+}$part of the wave with the amplitude of the forward-traveling radiating mode and the term in brackets with the negative-traveling one.

The power radiated into air is given by

$$
\begin{aligned}
\left(\frac{\Delta P}{P}\right)_{\substack{\text { radiated } \\
\text { into air }}} & \sum_{\substack{\text { even } \\
\text { odd }}} \int_{k\left(n_{2}^{2}-n_{3}^{2}\right)^{1 / 2}}^{n_{2} k} d \rho\left\{\left[\left|h^{+}(\rho, L)\right|^{2}\right.\right. \\
& \left.\left.+\left|h^{-}(\rho, 0)\right|^{2}\right] \frac{\int_{0}^{\infty}\left|\&^{a}\right|^{2} d x}{\int_{-\infty}^{\infty}\left|\&^{a}\right|^{2} d x}\right\} .
\end{aligned}
$$

The term involving the integration with respect to $x$ gives the fraction of the air mode radiated into the air. Furthermore, the boundary conditions require that

$$
\begin{aligned}
& h^{+}(\rho, z=0)=0 \\
& \Longrightarrow h^{+}=-\frac{1}{2 i \beta} \int_{0}^{z} H(\rho, \zeta) d \zeta \\
& h^{-}(\rho, z=L)=0 \\
& h^{-}=\left[\frac{1}{2 i \beta} \int_{L}^{z} \exp -2 i \beta \zeta H(\rho, \zeta) d \zeta\right] \exp 2 i \beta z
\end{aligned}
$$

Using the previous conditions and (32), (12), and (20), we get

$$
\begin{aligned}
& h^{+}(\rho, L)=\frac{k^{2}\left(n_{1}^{2}-n_{3}^{2}\right)}{4 i \omega \mu P} \phi_{+} A C_{r} \\
& h^{-}(\rho, 0)=\frac{k^{2}\left(n_{1}^{2}-n_{3}^{2}\right)}{4 i \omega \mu P} \phi_{-} A C_{r}
\end{aligned}
$$

where

$$
\phi_{+}=\phi_{+}(\beta, L)=\int_{0}^{L} f(z) \exp i\left(\beta-\beta_{0}\right) z d z
$$

and

$$
\phi_{-}=\phi_{-}(\beta, L)=\int_{0}^{L} f(z) \exp -i\left(\beta+\beta_{0}\right) z d z .
$$

Using (19) and (26) we can calculate $h^{+}(\rho, L)$, namely

$$
\begin{aligned}
\left|h^{+}(\rho, L)_{i}\right|^{2}= & \frac{k^{4}\left(n_{1}^{2}-n_{3}^{2}\right)^{2}\left|\phi_{+}\right|^{2} \kappa_{0}^{2}}{\left|\beta_{0}\right|\left[d+1 / \gamma_{0}+1 / \delta_{0}\right]\left(\kappa_{0}^{2}+\delta_{0}^{2}\right) \pi|\beta|} \\
& \cdot\left[\left(\cos \sigma d-F_{i} \sin \sigma d\right)^{2}+\frac{\sigma^{2}}{\rho^{2}}(\sin \sigma d\right. \\
& \left.\left.+F_{i} \cos \sigma d\right)^{2}+\left(1+\frac{\sigma^{2}}{\Delta^{2}} F_{i}^{2}\right) \frac{\Delta}{\rho}\right]^{-1}
\end{aligned}
$$

where $\kappa_{0}, \gamma_{0}, \delta_{0}$ refer to the zero-order-mode solutions for (15)-(18).

Similarly,

$$
\left|h^{-}(\rho, 0)_{i}\right|^{2}=\frac{\left|h^{+}(\rho, L)_{i}\right|^{2}\left|\phi_{-}\right|^{2}}{\left|\phi_{+}\right|^{2}} \text {. }
$$

Finally, to calculate the fraction of the air mode radiated into air, we use (20)-(22) 


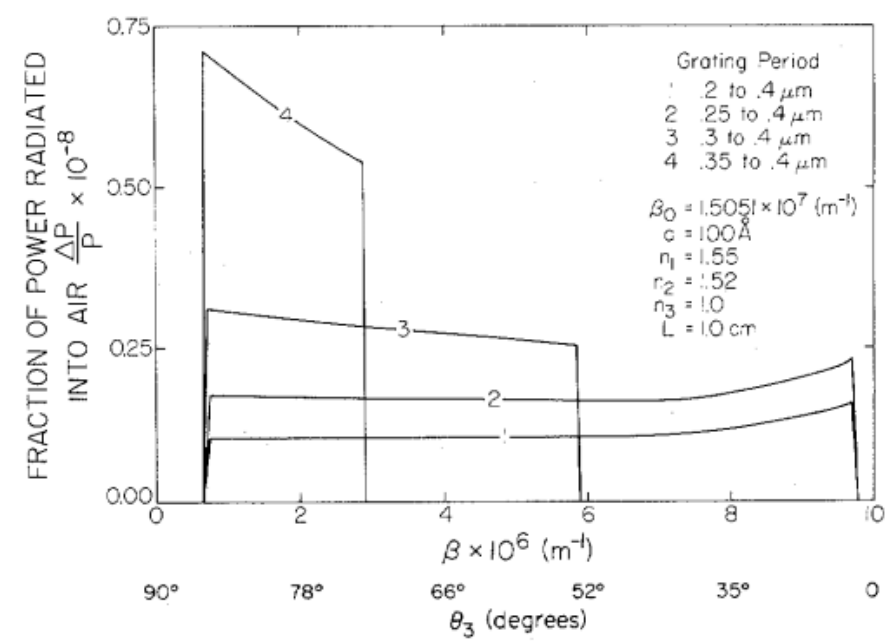

Fig. 9. Fraction of mode power radiated into air per unit $\beta$ as a function of $\beta$ or $\theta_{3}$, where $\theta_{3}$ is the angle of scattering with respect to the $z$ axis (see Fig. 6) for various chirps. The area under each curve represents the total power radiated into air for a given chirp.

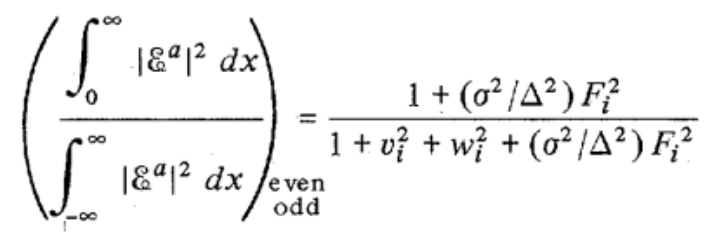

where

$$
\begin{aligned}
v_{i} & =\cos \sigma d-F_{i} \sin \sigma d \\
w_{i} & =\frac{\sigma}{c}\left(\sin \sigma d+F_{i} \cos \sigma d\right) .
\end{aligned}
$$

Now, using (45)-(43), (36) becomes

$$
\begin{aligned}
& \left(\frac{\Delta P}{P}\right)_{\substack{\text { radiated into } \\
\text { air per unit } \beta}}=\frac{S}{\rho}\left[\left|\phi_{+}\right|^{2}+\left|\phi_{-}\right|^{2}\right] \cdot \sum_{i=1}^{2} \\
& \cdot \frac{u_{i}}{\left[\left(v_{i}\right)^{2}+\left(w_{i}\right)^{2}+u_{i}\right] \cdot\left[\left(v_{i}\right)^{2}+\left(w_{i}\right)^{2}+\frac{\Delta}{\rho} u_{i}\right]}
\end{aligned}
$$

where

$$
u_{i}=1+\frac{\sigma^{2}}{\Delta^{2}} F_{i}^{2}
$$

and

$$
S=\frac{k^{4}\left(n_{1}^{2}-n_{3}^{2}\right) \kappa_{0}^{2}}{\left|\beta_{0}\right|\left(d+1 / \gamma_{0}+1 / \delta_{0}\right)\left(\kappa_{0}^{2}+\delta_{0}^{2}\right) \pi} .
$$

Equation (48) shows the fractional power radiated per unit beta for an arbitrary perturbation on the top surface. Once the perturbation is given, then $\phi_{+}$and $\phi_{-}$can be calculated.

For the particular case of the chirped grating with a transmission function given by (4), $f(z)$ can be written as

$$
f(z)=a \sin \left(\alpha z+\gamma z^{2}\right) .
$$

Direct substitution into (41) and (42) and using the method of stationary phase results in

$$
\left|\phi_{+}\right|^{2}=\frac{\pi a^{2}}{4 \gamma}
$$

if

$$
\gamma>0 \quad \alpha+\beta-\beta_{0}<0 \quad \alpha+\beta-\beta_{0}+2 \gamma L>0
$$

or

$$
\gamma<0 \quad \alpha+\beta-\beta_{0}>0 \quad \alpha+\beta-\beta_{0}+2 \gamma L<0
$$

or

$$
\gamma<0 \quad \alpha+\beta_{0}-\beta>0 \quad \alpha+\beta_{0}-\beta+2 \gamma L<0
$$

otherwise $\phi_{+}=0$.

Similarly,

$$
\left|\phi_{-}\right|^{2}=\frac{\pi a^{2}}{4 \gamma}
$$

if

$$
\gamma>0 \quad \alpha-\beta-\beta_{0}<0 \quad \alpha-\beta-\beta_{0}+2 \gamma L>0
$$

or

$$
\gamma<0 \quad \alpha-\beta-\beta_{0}>0 \quad \alpha-\beta-\beta_{0}+2 \gamma L<0
$$

or

$$
\gamma<0 \quad \alpha+\beta+\beta_{0}>0 \quad \alpha+\beta+\beta_{0}+2 \gamma L<0
$$

otherwise $\left|\phi_{-}\right|^{2}=0$.

These conditions, (52a)-(52c) and $(53 a)-(53 c)$, give the range of $\beta$ for which the guide radiates.

To illustrate (48), we present Fig. 9. The guide is $1.0 \mathrm{~cm}$ long, its thickness is $0.6425 \mu \mathrm{m}$, and the index of refraction is $n_{1}=1.55$. The substrate index of refraction is $n_{2}=1.52$, and that of air is taken to be $n_{3}=1.0$. The film perturbation is of the form of (51) and a was chosen to be $0.01 \mu \mathrm{m}$. The calculation for the fundamental mode gave $\beta_{0}=1.505 \times 10^{7}$ $\mathrm{m}^{-1}$ corresponding to a wavenumber of $9.78 \times 10^{6} \mathrm{~m}^{-1}$. The figure illustrates the fractional power output in the air per unit $\beta$ as a function of $\beta$ for various chirps. We see from the 


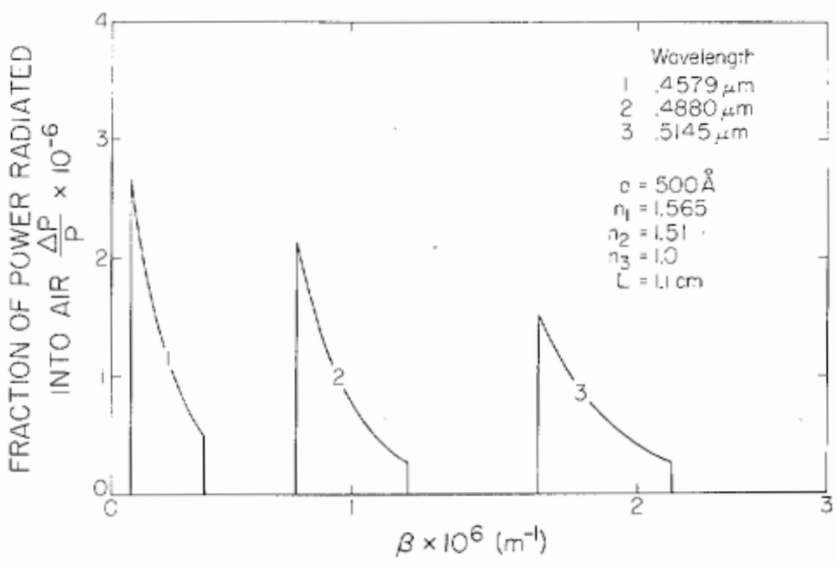

Fig. 10. Fraction of mode power radiated into air per unit $\beta$ as a function of $\beta$ for a chirp of $0.33-0.295 \mu \mathrm{m}$ and various wavelengths. The amplitude of the chirp is set at $500 \AA$, and the corrugation extends over a length $L$ of $1.1 \mathrm{~cm}$.

figure that the lower the chirp (curve 4) the narrower the range of $\beta$ distribution. In the limit of no chirp, we expect the familiar $\delta$-function. For high chirp we have a wide range of $\beta$ distribution extending over most of the theoretically possible range $\left(k_{z}=0\right.$ to $k$ ). The total power output radiated into air is the area under the curves. For Fig. 9, it ranges from 10 to 15 percent of the incident-mode power.

It is important to note that in the actual calculation the waveguide is divided into approximately one hundred sections. $\triangle P / P$ is calculated from the first section and then it is subtracted from the total $P$. This new value of $P$ is used as input power for the next section, and so on. This enables us to handle large power coupling and not be limited by the first Born approximation. This particular point is illustrated in Fig. 10. The total power output radiated into air is greater than 45 percent, due to the larger perturbation. In Fig. 10 the film thickness is $1.35 \mu \mathrm{m}$ and its index of refraction $n_{1}=1.565$. The substrate has an index of refraction $n_{2}=1.51$ and air $n_{3}=$ 1.0. The guide is $1.1 \mathrm{~cm}$ long and again the perturbation on the top surface is given by (51). In this case, $a$ is $0.05 \mu \mathrm{m}$, and the period varies from 0.295 to $0.33 \mu \mathrm{m}$. The different curves represent the fractional power output per unit $\beta$ for various wavelengths. It can be seen from this figure that different wavelengths radiate over different and nonoverlapping $\beta$ ranges.

In addition, we have calculated the fractional power (per unit $\beta$ radiated into air and substrate) and found that, as pre. dicted by the theory [9], it is twice as large as the one radiated into air.

\section{Experimental Results}

As a first demonstration of a device based on chirped gratings we chose a focusing output coupler. This grating coupler, with variable period, was corrugated on the surface of an optical waveguide, and was designated to focus the light a few centimeters away from the surface.

A layer of Corning 7059 glass which was sputtered on a glass substrate served as a waveguide. The 7059 glass was sputtered using Technics MIM Model 5.5 ion-beam etching machine, resulting in a layer of a refractive index of 1.565 and thickness uniformity of about 5 percent. For the focusing experiment we deposited a layer of thickness $1.35 \mu \mathrm{m}$ (as measured by a Sloan Dektak instrument).

Chirped gratings were fabricated on the surface of the waveguide as follows: a layer of undiluted Shipley AZ1350B photoresist was spin coated at $3600 \mathrm{rpm}$ on the waveguide. After prebaking, the photoresist was exposed to the interference pattern of a collimated laser beam with a converging beam. As detailed above, such interference pattern gives rise to chirped gratings. We used the $\lambda=4579 \AA$ line from an $\mathrm{Ar}^{+}$ laser, and under the following conditions $\theta=94.5, F=1.33$, $L=1.2 \mathrm{~cm}$, we obtained gratings with periods varying from 0.29 to $0.33 \mu \mathrm{m}$ over a distance of $1.2 \mathrm{~cm}$.

Typically the laser beam intensity was $0.6 \mathrm{~mW} / \mathrm{cm}^{2}$ (in each leg) and the exposure time used was $60 \mathrm{~s}$. Gratings of high efficiency were obtained using an AZ 303 developer and 10-s development time. It should be mentioned that the intensity of $\mathrm{Ar}^{+}$lasers is usually inferior to that of $\mathrm{He}-\mathrm{Cd}$ ones, and in our experiment we had to use a fringe-stabilization system in order to improve the peak-to-trough height of the photoresist gratings.

The photoresist was next postbaked under vacuum for 30 min, and the waveguide was ion-beam etched through the photoresist, at ion current density $0.1 \mathrm{~mA} / \mathrm{cm}^{2}$ and accelerating voltage of $1800 \mathrm{~V}$, for $30 \mathrm{~min}$. The sample was kept at an angle of $30^{\circ}$ with respect to the ion beam. The gratings thus fabricated in the glass had a peak-to-trough height of about $500 \AA$.

In the focusing experiment, we coupled light from an argon laser into the waveguide using a prism coupler. The light entering the corrugated section was focused outside the waveguide. The position of the focal point $\left(x_{f}, z_{f}\right)$ was measured experimentally for various lines of the argon laser. The experimental points are shown in Fig. 11, along with the theoretical predicted curve for this particular waveguide.

An output prism coupler was added at the end of the corrugated region. The light intensity which was coupled out was measured for two cases: a) light going through the corrugated region, and b) light going through a neighboring uncorrugated region. The ratio between the intensities in case a) and case b) was found to be $1: 10$. 


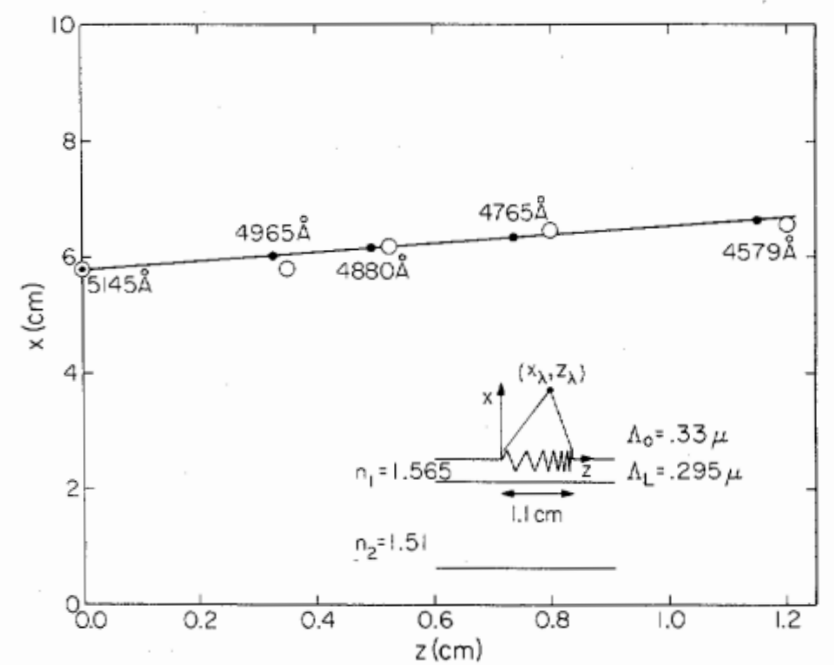

Fig. 11. Experimental and theoretical results of the focusing of the corrugated structure used. The solid line represents the theoretical position of the focus as a function of wavelength. The solid dots represent the focus of the prominent lines of the $\mathrm{Ar}^{+}$laser. The large circles are the experimental points for these wavelengths as measured with a two-dimensional translation probe.

\section{Discussion}

In this work we have demonstrated first how chirped gratings can be fabricated in a photoresist layer by the interference of a collimated beam with a converging beam. We have calculated how the chirping varies with the various parameters of the experimental setup involved. As one im. portant application of chirped grating, we demonstrated the focusing effect in a waveguide incorporating a chirped grating. The thickness of this waveguide and the chirp were chosen so as to focus the light about $6 \mathrm{~cm}$ away from the waveguide. The theoretical calculations, which were verified experimentally, show that the focal point moves by about $1.2 \mathrm{~cm}$ when the wavelength was changed from 4579 to $5145 \AA$. The chirped-grating structure therefore separates very well between propagating beams of different wavelengths, while focusing them outside the waveguide.

Other devices, which are based on chirped gratings, can also be realized. The first is a broad-band optical filter [10]. It is well known [12] that a grating structure with a period $\Lambda$ will act as a selective reflector, and will reflect only wavelength $\lambda$ that satisfies Bragg law $\lambda / 2 n=\Lambda$. The reflectivity can be rather high if the depth of the corrugations and if the length of the corrugated region are high. If the gratings have variable period $\Lambda(z)$, say between $\Lambda_{1}$ and $\Lambda_{2}$, then all the wavelengths $\lambda_{2}>\lambda>\lambda_{1}$ will be reflected, provided $\lambda_{1}=$ $2 n \Lambda_{1}$ and $\lambda_{2}=2 n \Lambda_{2}$. The response of this broad-band filter (i.e., reflectivity versus wavelength) can be changed by changing the chirp. Such broad-band filters may well be incorporated as reflectors in corrugated laser structures such as DFB lasers [3] or DBR lasers [4].

Another device, which may have a significant importance is a beam splitter. It was shown [10] that if a guided beam of wavelength $\lambda$ is incident on a corrugated region with an incidence angle $\alpha$, the beam will be deflected at an angle $2 \alpha$, provided $\lambda / 2 n \cos \alpha=\Lambda$, where $\Lambda$ is the period and $n$ is the refractive index of the waveguide. The fraction of the intensity that is deflected depends again on the corrugation depth and the length of the corrugated region. If the corrugated region consists now of chirped gratings with variable period $\Lambda(z)$, then a particular wavelength $\lambda_{1}$ will be reflected from a particular region where the period $\Lambda_{1}$ satisfies Bragg's law $\Lambda_{1}=\lambda_{1} / 2 n \cos \alpha$.

A different $\lambda_{2}$ will be reflected by $\Lambda_{2}$, so that $\Lambda_{2}=\lambda_{2} / 2 n$ $\cos \alpha$. Actually, $\lambda_{1}=\lambda\left(z_{1}\right)$ and $\lambda_{2}=\lambda\left(z_{2}\right)$, so that the two wavelengths will be reflected from different regions, and they will be separated spatially. And, therefore, if we have a beam that consists of many wavelengths $\lambda_{i}$, the chirped-grating structure will demultiplex it. It is conceivable by this method to demultiplex a signal traveling in a fiber, and to send each frequency component to a different fiber. If the directions of the previously mentioned beams are reversed, the chirped grating structure will act as a multiplexer. Beams of different frequencies will be reflected now to a common direction, and could then be focused into a single fiber. Such multiplexing and demultiplexing experiments are now in progress in our laboratory.

Chirped-gratings structures will undoubtedly be useful not only in integrated optics but in other fields. One important field is that of surface acoustic waves, where gratings with variable periods have been made using electron-beam writing [11].

\section{AC KNOWLEDGMENT}

The authors wish to thank Prof. N. George for many helpful discussions, and D. R. Armstrong and P. Agmon for their assistance.

\section{REFERENCES}

[1] A. Yariv and M. Nakamura, "Periodic structures for integrated optics," IEEE J. Quantum Electron., this issue, pp. 233-253.

[2] D. C. Flanders, H. Kogelnik, R. V. Schmidt, and C. V. Shank, "Grating filters for thin film optical waveguides," Appl. Phys. 
Lett., vol. 24, p. 194, 1974.

[3] K. Aiki, et al., "GaAs-GaAlAs distributed feedback diode lasers with separate optical and carrier confinement," Appl. Phys. Lett., vol. 27, p. $145,1975$.

[4] W. Ng, H. W. Yen, A. Katzir, I. Samid, and A. Yariv, "Room temperature operation of GaAs Bragg Mirror lasers," Appl. Phys. Lett., vol. 29, p. 684, 1976.

[5] M. L. Dakss, L. Kuhn, P. F. Heidrich, and B. A. Scott, "Grating coupler for efficient excitation of optical guided waves in thin films," Appl. Phys. Lett., vol. 16, p. 523, 1970.

[6] R. A. Bartolini, "Characteristics of relief phase holograms recorded in photoresists," Appl. Opt., vol. 13, p. 129, 1974.

[7] D. Marcuse, "Mode conversion caused by surface imperfections of a dielectric slab waveguide," Bell Syst. Tech J., vol.
48, p. $3187,1969$.

[8] —, Theory of Dielectric Optical Waveguides. New York: Academic, 1974

[9] W. Streifer, R. D. Burnham, and D. R. Scifres, "Analysis of grating-coupled radiation in GaAs:GaAlAs lasers and waveguides-II: Blazing effects," IEEE J. Quantum Electron., vol. QE-12, p. 494, 1976.

[10] H. Kogelnik, "Filter response of nonuniform almost-periodic structures," Bell Syst. Tech. J., vol. 55, p. 109, 1976.

[11] K. S. Pennington and L. Kuhn, "Bragg diffraction beam splitter for thin film optical guided waves," Opt. Commun., vol. 3, p. $387,1971$.

[12] R. C. Williamson, "Properties and applications of reflectivearray devices," Proc. IEEE, vol. 64, p. 702, 1976. 